\title{
SIGNIFICADOS Y PRÁCTICAS CULTURALES DE LA MENSTRUACIÓN EN MUJERES AYMARA DEL NORTE DE CHILE. UN APORTE DESDE EL GÉNERO A LOS ESTUDIOS ANTROPOLÓGICOS DE LA SANGRE MENSTRUAL
}

\author{
MEANINGS AND CULTURAL PRACTICES OF MENSTRUATION IN AYMARA \\ WOMEN IN NORTHERN CHILE. A CONTRIBUTION FROM A \\ GENDER PERSPECTIVE TO THE ANTHROPOLOGICAL STUDIES \\ OF THE MENSTRUAL BLOOD
}

María Belén Vásquez Santibáñez y Ana María Carrasco Gutiérrez²

\begin{abstract}
Este artículo busca aportar a un mayor conocimiento respecto de los significados que las distintas sociedades le dan a la sangre menstrual. Para dar cuenta de lo anterior, se realiza un recorrido histórico acerca de los estudios antropológicos que han profundizado respecto de esta temática y se presentan resultados de investigación que describen y explican las prácticas y significaciones culturales de la menstruación desde una perspectiva de género en tres generaciones de mujeres aymaras del norte de Chile, ahondando en las prohibiciones, creencias, tradiciones orales y tabúes sexuales propios de esta cultura.
\end{abstract}

Palabras claves: mujeres, género, menstruación, cuerpo, Aymara.

This article aims to contribute to a better understanding of the meanings that different societies give to menstrual blood. To achieve this, a historical overview of in-depth anthropological studies on the subject was carried out; in addition, research results which describe and explain cultural practices and meanings of menstruation are presented from a gender perspective in three generations of Aymara women in northern Chile, delving into prohibitions, beliefs, oral traditions and sexual taboos characteristic of this culture. Key words: Woman, gender, menstruation, body, Aymara.

El ciclo menstrual constituye una parte importante de la cotidianidad universal de las mujeres. Los conocimientos respecto de la menstruación, generalmente se relacionan con los significados del desarrollo biológico y cambios psicológicos del cuerpo femenino. Sin embargo, existen diversas formas culturales de construir los saberes que encauzan dicho fenómeno. De esta manera, la variabilidad cultural da cuenta del alcance que posee la menstruación, la significación que se le atribuye y el manejo corporal que mensualmente se tiene con el sangrado menstrual.

La disciplina antropológica, desde mediados del siglo XX, ha buscado profundizar en las representaciones y significados que se inscriben en la sangre menstrual, según cada entorno social en particular. Con ello, se ha demostrado la existencia de actitudes positivas y negativas asignadas a las significaciones menstruales determinadas por las diversas ideologías, religiones, cosmovisiones, etc. Desde estos paradigmas, se han efectuado estudios que han visibilizado la postura positiva de la sangre menstrual, recalcando las creencias mágico-religiosas y su asociación a la luna y al ciclo agrícola. Por otro lado, se han presentado investigaciones que distinguen los tabúes negativos acerca de las representaciones y las prácticas de la menstruación, subrayando la opresión y la marginación del cuerpo femenino, asî como también los símbolos culturales asignados a la toxicidad y peligrosidad de la sangre menstrual que se puede transmitir tanto a la comunidad como a las personas que son parte de ella. Los estudios que se realizan hoy permiten observar la transversalidad de la menstruación como una construcción sociocultural que posee símbolos, representaciones y prácticas que, mediante la articulación de los tabúes, los

\footnotetext{
1 Programa de Doctorado en Antropología UCN-UTA, Universidad Católica del Norte. Calle R. P. Gustavo Le Paige No 380, San Pedro de Atacama, Chile. belenvasquezs@gmail.com

2 Departamento de Antropología, Universidad de Tarapacá. Av. 18 de Septiembre No 2222, Arica, Chile. anycarrasco@gmail.com
} 
ritos y mitos, dan cuenta de aspectos analíticos relacionados a las temáticas de género, poder, identidad, etnicidad, entre otros.

La población aymara del extremo norte de nuestro país no está exenta de lo revelado. El haber vivenciado procesos sociohistóricos que han provocado cambios en sus modos y condiciones de vida, fue determinando que su población se enmarque dentro de significaciones específicas para el ciclo menstrual.

Los resultados de investigación, que presentamos en este artículo, buscan un acercamiento a este fenómeno observando el modo en que la cultura aymara impone significados y prácticas colectivas que moldean el pensar y accionar de los sujetos sociales. De esta manera, se indaga respecto de las prescripciones, prohibiciones, creencias, tradiciones orales y tabúes sexuales, propios de la población aymara, teniendo en cuenta el contexto sociohistórico, generacional y geográfico que actúan en definitiva como garantes de prácticas tradicionales o como gestores de nuevas formas de vivir el proceso menstrual.

Así, primero se analizarán algunos estudios realizados desde la disciplina antropológica que profundizan en las significaciones negativas de la sangre menstrual, las representaciones y prácticas menstruales relacionadas a la fertilidad, magia y estatus femenino; algunas investigaciones actuales enfocadas en conocer las significaciones de la sangre menstrual; y la revisión de estudios que sacan a la luz ritos, división sexual del trabajo, relaciones de género y sexualidad de la población aymara. Luego, se da cuenta por medio del análisis de los testimonios de las mujeres aymaras entrevistadas, de las prácticas y significados que poseen respecto de la menstruación; se analizan los conocimientos transmitidos familiarmente a las mujeres respecto del ciclo menstrual y la concepción de su propio cuerpo; se incorporan antecedentes respecto de influencia y contenidos valóricos que entregan los agentes externos y que intervienen en los conocimientos que poseen las mujeres aymara con el sangrado menstrual. Finalmente, se establecen algunas reflexiones sobre la influencia de la identidad de género aymara en los significados y prácticas socioculturales que recaen en el ciclo menstrual.

La metodología empleada ha sido del tipo cualitativa. La información contenida en este artículo se ha obtenido mediante la recolección de información primaria y secundaria. En el primer caso, se realizaron entrevistas estructuradas a 24 mujeres aymaras radicadas en la ciudad de Arica, XV Región de Arica y Parinacota, seleccionadas por criterios de edad y residencia urbana, fundamentalmente. Respecto de la condición étnica se utilizó como criterio principal el de autoadscripción, considerándose también identificación de sus pares, lugar de origen y apellidos. La variable edad remite tanto al ciclo vital, es decir, a etapas en la vida de cada entrevistada que define posiciones distintas tanto en la familia como en la sociedad en general, como también alude a generaciones y épocas culturales diferenciadas en la sociedad en su conjunto. Así, se distinguieron tres categorías de sujetos: jóvenes (15-25 años), adultos (26-45 años) y adultos mayores (46-65 y más años). Su análisis fue mediante el uso de software MAXQDA.

\section{Los Estudios Antropológicos de la Menstruación: Pasado y Presente}

\section{Primeras aproximaciones antropológicas sobre la sangre menstrual}

Los primeros estudios antropológicos para explicar la menstruación, su proceso y sus particularidades se evidencian a mediados del siglo XX. Serían estos aportes teóricos, por tanto, los responsables de producir una apertura al tema desde las definiciones puramente biológicas, propias de la época, hacia la idea de una concreta construcción sociocultural del proceso menstrual (cf. Alarcón 2005; Bobel 2010 [1963]; Buckley y Gottlieb 1988; Foster 1996; Gómez 2002; Lamas 1999; Rostworowski 2003; Steward y Strathern 2002; entre otros).

Las investigaciones que se han desarrollado desde entonces se han basado en determinar los significados y las prácticas culturales diversas que se establecen en torno a la menstruación. En un primer momento los estudios estuvieron dirigidos en los juicios negativos asignados a la menstruación y a cómo las relaciones de poder y género, así como también la dominación masculina, jugaban un papel simbólico sustancial en las sociedades indígenas. En esta línea, el estudio de los tabúes y los ritos religiosos articulados en la vida social de las comunidades cimentó las bases de las primeras investigaciones que dieron lugar a reflexiones respecto de los prejuicios de contaminación simbólica (polución) y de la sangre menstrual, a la segregación o al aislamiento 
de la mujer (Buckley 1982; Douglas 1973 [1966]; Lévi-Strauss 1988 [1955]; McGilvray 1982; Turner 1969 [1964]). Entre estos aportes destacan los estudios desarrollados en la antropología clásica de la década de 1960, que resultaron ser cruciales por la manera de representar la menstruación en las diversas sociedades, en donde se enfatizaba en los aspectos simbólicos de la sangre menstrual como una imagen negativa y esencialmente contaminante.

En años posteriores se desarrollan nuevas investigaciones etnográficas que fueron cruciales para continuar con esta perspectiva que consideraba a la menstruación como un acontecimiento esencialmente negativo (Buckley 1982; McGilvray 1982).

\section{Fertilidad, magia y estatus: la sangre femenina y su construcción sociobiológica}

A mediados de la década de 1980 nace un segundo momento, en el cual los estudios etnográficos comienzan a describir el proceso menstrual de manera positiva. En contraste a los tabúes y a sus representaciones, esta vez la menstruación como proceso sociobiológico comenzó a entenderse como poseedora de un carácter mágico, esencialmente singular, e incluso como productora de estatus femenino representado en su capacidad reproductiva (Douglas 1973 [1966]; Gómez 2010; Meigs 1984; Platt 2002; Reyes 2009; Strathern y Steward 1997; entre otros).

Aparecen también, en este momento, estudios que comparan las prácticas de sangre, tradicionalmente entendidas como la fuente de la fertilidad y parte fundamental del ciclo cósmico de la vida y la muerte, con nuevas concepciones en las que se le da una connotación contaminante esencial del cuerpo femenino. Afirmándose que este tipo de representaciones y prácticas se relacionan directamente con la influencia de género reproducida desde la época colonial, así como también con la visión del antropólogo/a que lleva consigo al campo y a sus investigaciones (Meigs 1984; Strathern y Steward 1997).

En la primera década del siglo XXI las investigaciones toman fuerza respecto de la capacidad reproductiva femenina; la importancia de la sangre menstrual en la gestación del feto; la complementariedad femenina y masculina representada por la sangre roja/menstruación/ femenina y sangre blanca/semen/masculina; y los aspectos positivos insertos en la construcción y cuidado del cuerpo de la mujer según sus valores del grupo social (Platt 2002; Reyes 2009).

\section{Representaciones y prácticas de lo menstrual en la esfera social: género, poder e identidades sexuales}

En la actualidad, los estudios en la materia se esfuerzan por articular los tabúes menstruales con ciertas categorías vinculantes a diversas dimensiones de la esfera social. Entre ellas se destaca, por ejemplo, la construcción del género y del poder en la vida social, y las identidades sexuales, con sus espacios de participación y funciones ligado a lo femenino. En definitiva, dicha profundización analítica en el estudio de los tabúes tiene por objeto buscar interpretaciones que se ajusten a la menstruación como un proceso plausible de ser un acontecimiento social y simbólicamente emblemático en toda colectividad humana (Freidenfelds 2009; Gómez 2010; Masquelier 2011; Napolitano 1997; Reyes 2009; Steward y Strathern 2002; Umeora y Egwuatu 2008).

Estudios antropológicos recientes, que buscan profundizar y encontrar respuestas plausibles acerca de las diferencias jerárquicas de los sexos, han incursionado en las representaciones propias de cada sociedad. Así, una forma de entrada ha sido "hurgar" en el imaginario humano, especialmente en aspectos relacionados con el cuerpo y los fluidos que segrega (Heritiér 2002:7). La materia prima de lo simbólico, que designaría las diferencias sexuales, sería el cuerpo, concebido como el primer lugar de observación de los datos sensibles, simples, presentes en cualquier cultura y que pueden dar evidencias elementales. En todas las sociedades, la diferencia sexual se observaría bajo un lenguaje binario y jerarquizado que radica en una característica simbólica y desigual del cuerpo femenino, la sangre. Líquido presente en ambos cuerpos, pero que en la mujer es derramada involuntariamente (menstruación), no por libre arbitrio como puede ocurrir en los hombres (luchas) (Heritiér 2002:232). Este simbolismo sería el que construye los géneros dando sentido a las oposiciones binarias y, por ende, a las prácticas socioculturales.

En consecuencia, el interés de las investigaciones que se han realizado en las últimas décadas han centrado su quehacer en comprender el proceso menstrual como un acontecimiento social y cultural articulado en función de relaciones sociales de 
género que determinan el sentido de las mismas. Así, es posible considerar la menstruación como el resultado de valores y significaciones que se instituyen según su propia construcción del mundo; comprender tales representaciones en su diversidad es una de nuestras preocupaciones. Como señala Bourdieu (2000 [1998]), existe una construcción social arbitraria de lo biológico, de los cuerpos y de los géneros, que se posiciona como algo natural, generalmente bajo una postura androcéntrica de la división sexual que guía la vida social de los individuos. Teniendo en cuenta estos antecedentes, sería un error seguir subvalorando la menstruación como dimensión de análisis, y en este sentido, creemos que conocer detalles socioculturales como las representaciones y los significados propios de la tradición aymara son, sin duda, datos valiosos que amplían los conocimientos investigativos y que sirven de apertura a nuevos campos de estudio.

\section{Reflexiones Acerca del Significado del Ciclo Menstrual en Mujeres Aymaras del Norte de Chile y su Relación con las Construcciones de Género}

En nuestro país, el estudio de la menstruación constituye un área de investigación muy poco abordada desde la disciplina antropológica. No obstante, poco a poco la historia de la religión y la antropología han reforzado paralelamente los conocimientos respecto del tema y se han insertado como nuevas alternativas de análisis para comprender el proceso natural y la carga simbólica que atañe al mismo.

En lo que respecta a la población aymara del extremo norte de Chile, existe gran cantidad de información relacionada con los procesos históricos que ha afectado su sociedad y su cultura, tales como el proceso de chilenización, influencia judeocristiana, entre otras, generando cambios en sus modos y condiciones de vida. Las principales transformaciones experimentadas tienen su origen desde mediados del siglo XIX, en lo referido a su vínculo con las ciudades, con la desarticulación de las comunidades históricas campesinas dando paso a “(...) pequeñas localidades agrarias de los valles y a las comunidades sucesoriales de las tierras de la alta cordillera (...)" (Gundermann 2003:57). Este fenómeno toma fuerza a partir de 1940 y 1950 en donde, en primera instancia, al desencadenarse la crisis salitrera el campesinado andino de los valles bajos busca nuevos rumbos. Uno de los aspectos que ha tenido mayor incidencia en los procesos de transformaciones y cambios es la migración, que se torna masivo a partir del auge económico que experimentaron las ciudades de Arica durante 1950 e Iquique a mediados de 1970 (cf. Carrasco y González 2012; Gavilán 2002; González 1995, 1996; González et al. 2014; Grebe 1986; Gundermann 2001; Gundermann et al. 2014).

Es preciso mencionar que en la zona norte de Chile no existen estudios centrados específicamente en la problemática que nos ocupa. Sí es posible mencionar una serie de investigaciones que abordan fundamentalmente aspectos económicos, laborales y/o sociales de las mujeres aymaras, vinculándose a nuestros objetivos mediante sus aportes respecto de la identidad de género, dando luces para distinguir las significancias en torno al ciclo menstrual femenino aymara (cf. Carrasco 1998, 2003; Carrasco y Gavilán 1992, 2005, 2009; Gavilán 1995, 1999, 2005).

Asimismo, debemos reconocer el valor de las investigaciones realizadas en países como Perú y Bolivia respecto de la población aymara en temáticas como la cosmovisión, vida reproductiva y religiosidad andina, ya que su emplazamiento histórico nos permite rescatar conocimientos que dan lugar a las concepciones del sexo, género y cuerpo y a la representación cultural que le otorgan a lo masculino y femenino. Lo anterior, teniendo presente que los focos de análisis pueden variar en el contexto país, de uno y otro (Albó 1987; Arnold 1997, 2006; Arnold y Yapita 1996, 1998, 1999; Cadorette 1977; Grebe 1981; Montes 1984; Platt 2002; van Kessel 1993).

Un aspecto primordial en el análisis de la transmisión de significados y prácticas del ciclo menstrual es el proceso de socialización que recae en la familia de origen, la parentela bilateral y en algunos espacios rurales, la comunidad de la cual son parte los individuos que se adscriben a la población aymara. Esto porque permite entender cómo a través de la reproducción y/o transformación de las prácticas familiares formamos parte de estructuras socioculturales que confluyen cotidianamente en los significados menstruales. En este sentido, las diferencias etáreas de las mujeres aymara, que conformaron nuestros casos de estudio, nos permitieron descubrir la presencia de contenidos valóricos que difieren en la fuerza con la que la familia transmite los significados y prácticas del 
ciclo menstrual. En términos generales, podemos decir que entre las mujeres jóvenes y adultas aymara existe una mayor transmisión de saberes traspasados por los agentes externos (establecimientos educacionales y de salud, los pares, medios masivos de comunicación y la influencia judeocristiana). Así, no se presentan conductas o modos de actuar con la sangre menstrual que demuestren una tradición familiar aymara. Para el caso de los significados del ciclo menstrual, observamos una combinación de agentes internos y externos que están configurando los significados de este acontecimiento. Sin embargo, la influencia de la familia solo se percibe en algunos de los testimonios de las mujeres jóvenes y adultas aymara. En este sentido, la importancia que se desprende de los agentes externos en ambas generaciones abarca la mayor parte y solidez de los testimonios, siendo los ejes centrales que moldean las experiencias femeninas de la menstruación.

En mi familia no hablaban ese tema para nada, de hecho yo, me llegó la regla y yo no sabía, porque de hecho para mí la regla no existía, ni si quiera me prepararon para eso, a mi me llegó la regla e inclusive me enfermé estuve hospitalizada (Mujer $N^{\circ} 11,33$ años).

La importancia biomédica que toma lugar entre los padres de las mujeres aymara de estos rangos etáreos genera que el proceso de transmisión de conocimientos sea principalmente mediante establecimientos educativos y el sistema de salud formal. Bajo dichos saberes se manifiestan los significados y prácticas que las mujeres le atribuyen al sangrado menstrual; por una parte, las ideas que dan cuenta de la privacidad e intimidad del acontecimiento, la predisposición positiva a los cambios físico-emocionales, el concebir la menarquia como el paso de niña a mujer, y la imagen femenina con capacidades reproductivas y fértiles $\mathrm{y}$, por otra, las prácticas que exhiben los cuidados que las mujeres deben mantener para evitar embarazos y con el cuerpo en relación con la vestimenta e higiene personal.

Fue sorpresa porque dije 'esto que es', o sea, quedé sorprendida, me acuerdo que un día llegué corriendo a la casa porque estaba con sangre y todo y yo no tenía idea de nada porque mi mamá tampoco nunca me dijo nada (...) mi mamá nunca me comentó ninguna cosa, no, nunca, no (Mujer $\mathrm{N}^{\circ} 13,32$ años).

A diferencia de lo que ocurre entre estos grupos, debemos distinguir la importancia familiar que recae en la adopción de conocimientos acerca de la menstruación entre las mujeres adultas mayores, asociado a prácticas del cuidado del cuerpo y representación de la sangre menstrual. A pesar de que la transmisión de conocimientos familiares no sea parte del total de las entrevistadas, destaca en este grupo la fuerza que adquiere en algunos testimonios los saberes, significaciones y prácticas de la menstruación. Esto alude a algunos de los componentes que rescatan Carrasco y Gavilán (1992, 2009) en sus investigaciones, dando cuenta que existen vínculos con ciertas creencias o elementos simbólico-religiosos que han influido en los significados atribuidos a la sangre menstrual. En esta línea, y como ha sido señalado, predominan ejemplos simbólicos de la Pachamama (tierra), Phaxsi (luna), Mallkus y T'allas (los seres de los cerros, de las vertientes y de las pircas), que forman parte integral del conjunto de significados, asociados a la capacidad reproductiva femenina aymara, que poseen significaciones de fertilidad y reproducción relacionadas al cuerpo femenino. Una de ellas es la relación de la capacidad fértil de la mujer y la luna; se concibe que solo al término de la menstruación la mujer aymara alcanza su mayor capacidad reproductiva que, a la vez, está acompañada de la luna nueva. De esta manera, se explica una relación simbólica de etapas entre el ciclo menstrual y lunar en donde la ovulación corresponde a la fase luna nueva, la premenstruación al cuarto creciente, la menstruación a la luna llena y la preovulación al cuarto menguante. Otro ejemplo es la fuerza y vitalidad que adquiere la sangre para dicha población y que debe traspasar a la tierra o Pachamama, Mallkus y T'allas contribuyendo a la fertilidad de la comunidad; en este sentido, podemos deducir que sobre la base de los aspectos simbólicos de seres o deidades, se le otorga a la sangre menstrual la fuerza vital para la reproducción del grupo social. Estas creencias nos permiten concebir que entre las mujeres adultas mayores aymara aún se mantienen presentes creencias simbólicas y religiosas que les ayudan a comprender aspectos relacionados con su rol femenino, su cuerpo, y su propia sexualidad. 
A mi parecer yo creo que uno no puede tener relaciones sexuales durante la regla, pero parece que si lo hacen. Yo creo que estando con la regla la mujer se puede dañar, se puede enfermar (Mujer $N^{\circ} 17,52$ años).

Cuando la mujer termina de menstruar, después ahí molestan hombres, ahí si vuelven y wawa (...) ahí sí (...) (Mujer $\mathrm{N}^{\circ} 23,92$ años).

De esta forma, concebimos que la hegemonía corporal que atañe a las generaciones jóvenes no intervino en la concepción menstrual del total de las mujeres adultas mayores aymara. A pesar de que muchas de ellas no obtuvieron los saberes de manera directa de sus familiares, el cotidiano sociocultural les ayudó a comprender el cuerpo femenino como un conjunto de sensaciones que emerge y se asimila a la naturaleza y la sangre menstrual como un hecho que otorga fertilidad a la sociedad.

Por otro lado, percibir la menstruación como la transición de niña a mujer es uno de los significados que son transmitidos familiarmente y que es transversal entre los distintos grupos etarios. Se plantea que desde la llegada de la menarquia el cuerpo femenino posee capacidades fértiles y reproductivas que les permite a las mujeres ser madres, significancia que entrega resultados decisivos respecto del cómo están comprendiendo el rol reproductivo femenino. Sumado a ello, es interesante rescatar entre las mujeres jóvenes y adultas aymara la reproducción de la "intimidad" del cuerpo femenino y de la menstruación como proceso natural, dando cuenta que la privacidad es naturalizada en algunos testimonios, y que se expresa como una importante representación asociada a la construcción social del cuerpo que da lugar a la posibilidad de concebir la reproducción humana. Sin embargo, resulta necesario recalcar que en los testimonios de las mujeres adultas mayores aymara se rescata el carácter natural de la sangre menstrual que busca transmitir la importancia de la menstruación a las generaciones más jóvenes.

Sabes que realmente no me acuerdo, porque no sentí dolores, no sentí nada, si lo único que sé es que de la noche a la mañana desperté y tenía manchada, y mi mamá recién me dijo que yo tenía que tener un ciclo, como toda mujer normal que evoluciona, que iba a menstruar una vez al mes y que tenía que ver, ocuparme yo de ver cuántos días me duraba eso, y eso fue todo. No me asusté mucho, no tenía idea porque mi mamá nunca me había dicho nada. Mi vieja en ese sentido fue súper (...) no me habló de menstruación, no me habló ni si quiera de lo que es sentir atracción por otra persona, de sexo no se habló nunca en mi casa (...) (Mujer $\mathrm{N}^{\circ} 16,51$ años).

La mujer si, el ganado, pasteando (...) lavar hay, antes no se conocían ni pañales, con pollera no ma, falda, harta falda (...) ahí no más estamos andando, antes no conocía ni calzón (...) manchar caminando (...) da miedo cuando la gente hay visitar, da miedo (...) a veces poquito (...) no hay que lavarse (...) (Mujer $\mathrm{N}^{\circ}$ 23, 92 años).

Si bien se planteó la escasez de investigaciones que rescaten la importancia que posee el ciclo menstrual en la población aymara del norte de Chile, los estudios en materias de género, rituales y de división sexual del trabajo han sido concluyentes para vislumbrar la construcción del cuerpo en el ser hombre y ser mujer aymara. Por esta razón es pertinente, en nuestro estudio, reflexionar en torno al género, núcleo central que se reproduce de generación en generación y que se impregna en cada una de las significaciones del ciclo menstrual aymara. De esta forma, con el transcurso de las generaciones la menstruación da cuenta de normas y éticas culturales en sus significados y prácticas que orientan las significaciones adscritas a esta población. Sin embargo, resulta indispensable aclarar que, entre los testimonios de las mujeres adultas mayores, se acentúan los saberes familiares y tradicionales respecto de la concepción de la sangre menstrual.

La construcción de género es esencial para visualizar las normas sociales establecidas en cada sexo y que se sostienen como modelos conductuales familiares para entablar relaciones sociales con los demás. Como plantea Francoise Héritier (1996), en todas las sociedades la diferencia sexual se observa bajo un lenguaje binario y jerarquizado que radica en una característica simbólica y desigual del cuerpo femenino; de esta forma, el origen simbólico sería el que construye los géneros dando sentido a las oposiciones binarias $\mathrm{y}$, por tanto, a las prácticas socioculturales. 
De esta forma, durante el ciclo de vida de la mujer aymara se idealizan los comportamientos, se diferencian de acuerdo con el género, y sufren procesos de transformación en el desarrollo de las distintas etapas de la vida. Así, durante la adolescencia comienza un entrenamiento hacia la vida adulta por medio del desarrollo individual y la adquisición de una mayor autonomía, además de vivenciar la menarquia como un acontecimiento que orientará los comportamientos y pautas de la mujer aymara.

La familia ha sido una de las instituciones que ha tenido un papel fundamental en la reproducción de las identidades de género aymara. Este tipo de representaciones históricas inconscientes se encuentran inscritas en el lenguaje y en los significados que contiene la menstruación que, por lo demás, en muchos casos está relacionada con aspectos simbólicos negativos o emociones corporales, como sentimientos de miedo, vergüenza, timidez, entre otras.

\section{(...) algunos nada decían (...) papás decían nada pasa (...) por eso algunas, por eso mujeres asustaban, deprimían y mataban, si (...) por eso a mí abuelos dijeron nada pasa, es normal mijita, de la mujer, la sangre (...) normal decían (...) (Mujer $\mathrm{N}^{\mathrm{o}} 23,92$ años).}

No podemos dejar de lado que en las raíces de la institución familiar se percibe fuertemente el peso de la tradición judeocristiana. Como ya ha sido mencionado, las mujeres poseen imposiciones específicas de la ideología cristiana; principalmente, en cuanto a las maneras de comportarse frente a la sociedad y sanciones específicas que falten la pureza de la mujer. El Antiguo Testamento de la Biblia, específicamente el apartado del Levítico (caps. 11-16), hace referencia a las impurezas que posee el cuerpo de la mujer a causa de la menstruación. Esta es concebida como la inmundicia que posee la mujer una vez al mes, que puede ser transmitida a cualquier cosa u objeto y que, bajo estas leyes, demuestra la discriminación hacia el género femenino.

(...) Si un hombre se acuesta con una mujer durante su derrame menstrual y descubre su desnudez, ha descubierto, y ella también, la fuente de su sangre; ambos serán borrados de en medio de su pueblo (...) La mujer que ha tenido sus reglas será impure por espacio de siete días, por ser un derrame de sangre de su cuerpo. Quien la toque será impuro hasta la tarde (...) Si un hombre se acuesta con ella a pesar de su impureza, comparte su impureza y queda impuro siete días; toda cama en que él se acueste sera impura (...) (Lev., cap. 15).

Desde esta perspectiva, la mujer aymara ha permanecido indiscutiblemente en la vereda invisible; de esta forma, se ha buscado la manera de justificar la privacidad de su cuerpo mediante la fuerza que posee la identidad femenina y que conlleva ciertas actitudes y comportamientos morales. Así, se señala los hábitos, costumbres y pudores que deben poseer las mujeres aymara y que se deben conservar con el paso del tiempo. Sin embargo, y por los motivos ya mencionados, entre ellas se reproducen patrones asociados a aspectos biológicos, religiosos o transmitidos por los medios masivos de comunicación que crean ideales de género, justifican las bases de la familia y fijan maneras de comportarnos con nuestro cuerpo.

\section{Reflexiones Finales}

Los resultados de investigación presentados en este artículo se orientaron a conocer los significados y prácticas en torno a la menstruación de las mujeres aymara del extremo norte de Chile, dando cuenta de las imposiciones de ciertas prescripciones sociales, prohibiciones, creencias, tradiciones orales y tabúes sexuales. Buscando con esto contribuir en la construcción de nuevos saberes acerca del ciclo menstrual, las identidades de género, la sexualidad y corporalidad de las mujeres aymara.

En primer lugar, se percibe la influencia que posee la identidad de género en la determinación de dinámicas socioculturales, significancias del ciclo de vida de la mujer aymara y diferencias corpóreas de los sujetos. Asimismo, se observan las particularidades atribuidas a la sangre menstrual en la cultura aymara, entendida como la esencia de la fertilidad femenina, el momento oportuno para la procreación y la reproducción de los individuos. Dichos saberes relacionados a la sexualidad, construcción de género, división sexual del trabajo, economía tradicional y el cuidado del cuerpo, permiten dar cuenta de las diversas maneras de significar la sangre menstrual como una construcción cultural. 
Por los testimonios de nuestras entrevistadas se pueden encontrar resultados de orden generacional, dando cuenta que tanto la percepción como los procesos vividos descritos en cada testimonio presentan una marcada diferenciación entre las experiencias y significaciones del ciclo menstrual.

En relación con los conocimientos valóricos transferidos por la familia y otros agentes formales de socialización, vemos que las mujeres jóvenes aymara adquieren saberes antes y durante la menarquia en los establecimientos educacionales, docentes, amistades y agentes de salud y, solo en algunas ocasiones, de sus madres. En el caso de las mujeres adultas, la mayor parte de ellas posee una carencia de conocimientos de lo menstrual provocando sentimientos de temor al no comprender los cambios de su cuerpo. Finalmente, gran parte de los testimonios de las mujeres adultas mayores concuerdan en la escasez de conocimientos que obtuvieron respecto de la sangre menstrual, y solo algunas de ellas señalan a la familia como único transmisor de saberes respecto de la menstruación. Así, mientras las mujeres más jóvenes poseen una mayor incidencia de la vida urbana y de la biomedicina por medio de las instituciones educacionales y de salud, las generaciones adultas adquieren influencia por parte de la familia nuclear y de la ideología judeocristiana.

En cuanto a las significaciones de la sangre menstrual, se percibe que tanto la mujer joven, adulta y adulta mayor aymara relacionan, en mayor o menor grado, la llegada del ciclo menstrual con el inicio de la capacidad reproductiva femenina, el paso de niña a mujer, cambios físicos del cuerpo de la mujer y mayores cuidados relacionados a las relaciones sexuales e higiene del cuerpo femenino. Sin embargo, se observan diferencias notables al momento de significar la corporalidad femenina; de esta forma, entre las jóvenes aymara existe una fragmentación del cuerpo aislando las sensaciones de lo menstrual y privando las experiencias de este.
A diferencia de ello, distinguimos que las mujeres adultas mayores engloban las prácticas corporales como un todo que relaciona los acontecimientos físicos con el alma y la naturaleza de los sujetos. De esta manera, podemos decir que existe una construcción corporal que ha tendido a cambiar con el paso del tiempo a base de una regulación moral y dominación de normas establecidas en el cotidiano social.

Por otro lado, y en relación con las prácticas de la menstruación, tenemos que los cuidados físicos del cuerpo de las mujeres jóvenes están asociados mayormente a las imposiciones biomédicas que se han instaurado mediante el mercado, de las instituciones educacionales y de salud. A diferencia de ello, entre las mujeres adultas mayores la mayor parte de las prácticas tiene relación con el cuidado e higiene del cuerpo, así como por la naturalidad física con la cual enfrentan los días menstruales.

Como pudimos observar, existe una determinación sociocultural que sobre la base de los agentes internos y externos construyen simbolizaciones específicas atribuidas a la sangre menstrual. La familia aymara por medio de la figura de las madres, hermanas y tías cercanas transmiten cotidianamente los conocimientos y cuidados que deben mantener las mujeres con su cuerpo durante la menstruación. Este tipo de saberes se entremezclan de explicaciones biológicas y religiosas que definen el proceso y las precauciones que deben comenzar a guardar para y con su cuerpo.

Agradecimientos: Este artículo forma parte del Proyecto Fondecyt $N^{\circ} 1140022$; asimismo cuenta con el apoyo del Convenio de Desempeño Universidad de Tarapacá - Mineduc. De igual forma, agradecemos las sugerencias aportadas por los evaluadores, que sin duda han ayudado a mejorar este artículo. 


\section{Referencias Citadas}

Alarcón, M.A. 2005. Algunas consideraciones antropológicas y religiosas alrededor de la menstruación. Revista Colombiana de Obstetricia y Ginecología 56:35-45.

Albó, X. 1987. Formación y evolución de lo aymara en el espacio y el tiempo. Coloquio Estado y Región en los Andes, pp. 29-43. CBC, Cusco.

Arnold, D. 1997. Introducción. En Más allá del Silencio. Las Fronteras de Género en los Andes, compilado por D. Arnold, pp. 13-52. CIASE/ILCA, La Paz.

Arnold, D. 2006. Hacer al hombre a imagen de ella: aspectos de género en los textiles de Qaqachaka. Chungara 26:79-114.

Arnold, D. y J. Yapita 1996. Los caminos de género en Qaqachaka: saberes femeninos y discursos textuales alternativos en los andes. En Ser Mujer Indígena, Chola o Birlocha en la Bolivia Postcolonial de los Años 90, editado por S. Rivera Cusicanqui, pp. 303-392. Ministerio de Desarrollo Humano, La Paz.

Arnold, D. y J. Yapita 1998. Río de Vellón, Río de Canto. Cantar a los Animales, Una Poética Andina de la Creación. Colección Academia No 8, Hisbol e ILCA, La Paz.

Arnold, D. y J. Yapita 1999. Vocabulario Aymara del Parto y de la Vida Reproductiva de la Mujer. Family Health International e ILCA, La Paz.

Bobel, C. 2010 [1963]. New Blood. Third-Wave Feminism and the Politics of Menstruation. Rutgers University Press, New Brunswick, New Jersey y Londres.

Bourdieu, P. 2000 [1998]. La Dominación Masculina. Editorial Anagrama, Barcelona.

Buckley, T. 1982. Menstruation and the power of yurok women: Methods in Cultural Reconstruction. American Ethnologist 9:47-60.

Buckley, T. y A. Gottlieb 1988. Blood Magic. The Anthropology of Menstruation. University of California, California.

Cadorette, R. 1977. Perspectivas mitológicas del mundo aymara. Allpanchis 10:115-136.

Carrasco, A.M. 1998. Constitución de género y ciclo vital entre los aymaras contemporáneos del norte de Chile. Chungara 30:87-103.

Carrasco, A.M. 2003. Diferencias de género en las ideas y en las prácticas a través del curso de la vida entre los aymarás del norte de Chile. Taller de Estudios Andinos, Documentos de Trabajo, Arica.

Carrasco, A.M. y V. Gavilán 1992. Representaciones del cuerpo, sexo y género entre los aymara del norte de Chile. Chungara 41:83-100.

Carrasco, A.M. y V. Gavilán 2005. Sexualidad y género: la unidad de lo femenino y lo masculino como símbolos de reproducción y fertilidad entre los Aymará del norte de Chile. En Imaginarios, Identidades e Historias. Miradas desde la Antropología del Género, editado por L. Rebolledo, P. Tomic y E. Garduño, pp.169-180. Centro de Estudios Culturales de las Universidad Autónoma de Baja California, California.

Carrasco, A.M. y V. Gavilán 2009. Representaciones del cuerpo, sexo y género: una aproximación a las diferencias sexuales entre los aymaras del norte de Chile. Chungara Revista de Antropología Chilena 41:83-100.

Carrasco, A.M. y H. González 2012. La movilidad poblacional aymara en tiempos de postcomunalidad. Actas XXXIV Convegno Internazionale di Americanistica, Perugia.

Douglas, M. 1973 [1966]. Pureza y Peligro. Un Análisis de los Conceptos de Contaminación y Tabú. Siglo Veintiuno Editores, Madrid.

Foster, J. 1996. Menstrual time: The sociocognitive mapping of "the menstrual cycle". Sociological Forum 11:523-547.

Freidenfelds, L. 2009. The Modern Period. Menstruation in Twentieth-Century America. The Johns Hopkins University Press, Baltimore.

Gavilán, V. 1995. Aproximación a las relaciones de género entre los aymara del norte de Chile. En Temas Regionales $\mathrm{N}^{\circ} 3$, Corporación Norte Grande, Arica.

Gavilán, V. 1999. Elaboraciones de género en la religiosidad de mujeres y hombres del norte de Chile. Avances de Investigación. Ciencias Sociales 8:65-82.

Gavilán, V. 2002. "Buscando vida...”: Hacia una teoría aymara de la división del trabajo por género. Chungara Revista de Antropología Chilena 34:101-117.

Gavilán, V. 2005. Representaciones del cuerpo e identidades étnicas y de género. Estudios Atacameños 30:65-87.

Gómez, M. 2002. Representaciones y prácticas en torno a la menstruación y menarca entre las mujeres tobas: entre la salud de las mujeres y la construcción social del género femenino. Manuscrito en posesión del autor.

Gómez, M. 2010. La luna y la feminidad entre los tobas del oeste formoseño (Gran Chaco, Argentina). Artigos 11:47-64.

González, H. 1995. Los migrantes aymaras en la ciudad: Acceso a educación, vivienda y salud. Serie Documentos de Trabajo. Corporación Norte Grande, Arica.

González, H. 1996. Características de la migración campociudad entre los aymaras del norte de Chile. Serie Documentos de Trabajo. Corporación Norte Grande, Arica.

González, H., H. Gundermann y J. Hidalgo 2014. Comunidad indígena y construcción histórica del espacio entre los aymara del norte de Chile. Chungara Revista de Antropología Chilena 46:233-246.

Grebe, M.E. 1981. Cosmovisión aymara. Revista de Santiago 1:61-79.

Grebe, M.E. 1986. Migración, identidad y cultura aymará: puntos de vista del actor. Chungara 16-17:205-223.

Gundermann K., H. 2003. Sociedades indígenas, municipio y etnicidad: La transformación de los espacios políticos locales andinos en Chile. Estudios Atacameños 25:55-77.

Gundermann, H., H. González y J. Durston 2014. Relaciones sociales y etnicidad en el espacio aymara chileno. Chungara Revista de Antropología Chilena 46:397-421. 
Héritier, F. 1996. Masculino/Femenino. El Pensamiento de la Diferencia. Editorial Ariel, Barcelona.

Lamas, M. 1999. Usos, dificultades y posibilidades de la categoría género. Papeles de Población 21:147-178.

Levi-Strauss, C. 1988 [1955]. Tristes Trópicos. Ediciones Paidós Ibérica, Buenos Aires.

Masquelier, A. 2011. The bloodstain: spirit possession, menstruation, and transgression in Niger. Ethnos 76:157-182.

McGilvray, D. 1982. Sexual power and fertility in Sri Lanka: batticaloa tamils and moors. En Ethnography of fertility and birth, pp. 25-73. Academic Press Inc., Londres.

Meigs, A. 1984. Food, Sex and Pollution: a New Guinea Religion. Rutgers University Press, New Brunswick, New Jersey.

Montes, F. 1984. La Máscara de Piedra. Simbolismo y Personalidad Aymaras en la Historia. Editorial Quipus, La Paz.

Napolitano, V. 1997. Becoming a 'mujercita': Rituals, fiestas and religious discourses. The Journal of the Royal Anthropological Institute 3:279-296.
Platt, T. 2002. El feto agresivo. Parto, formación de la persona y mito-historia en los Andes. Estudios Atacameños 22:127-155.

Reyes, G. 2009. El cuerpo como unidad biológica y social: Una premisa para la salud sexual y reproductiva. Revista Colombiana de Antropología 45:203:223.

Rostworowski, M. 2003. Los genitales femeninos en la iconografía andina prehispánica. Psicoanálisis 3:127:138.

Steward, P. y A. Strathern 2002. Power and placement in blood practices. Ethnology 41:349-363.

Strathern, A. y P. Steward 1997. Sorcery and sickness: spatial and temporal movements in Papua New Guinea and Australia. Discussion Papers Series 1:1-27.

Turner, V. 1969 [1964]. La Selva de los Símbolos. Aspectos del Ritual Ndembú. Siglo XXI, Madrid.

Umeora, O. y V. Egwuatu 2008. Menstruation in rural Igbo women of south east Nigeria: Attitudes, beliefs and practices. African Journal of Reproductive Health 12:109-115.

Van Kessel, J. 1993. Pachamama, La Virgina: La que Creó el Mundo y Fundó el Pueblo. CIDSA, Puno. 